Pacific Journal of Mathematics

A HOM-FUNCTOR FOR LATTICE-ORDERED GROUPS 


\title{
A HOM-FUNCTOR FOR LATTICE-ORDERED GROUPS
}

\author{
JoRge MARTINEZ
}

\section{Results are presented that characterize subdirect products of reals (respectively, integers) functorially.}

By defining a quasi-order on the lattice-homomorphisms (henceforth: l-homomorphisms) of one abelian lattice-ordered group (henceforth: l-group) to another, one can set up a co-compatible system of partially ordered groups (henceforth: p. o. groups). Their co-limit $L(A, B)$, where $A$ and $B$ are the l-groups in question, is a directed, semi-closed p. o. group. If $A$ is a totally ordered group (henceforth: o-group) then $L(A, B)$ is simply the subgroup of Hom $(A, B)$ generated by the o-homomorphisms. On the other hand, if $B=R$, the additive group of real numbers with the usual order, then $L(A, B)$ is a cardinal sum of copies of $\boldsymbol{R}$, one for each maximal $l$-ideal of $A$. In general the co-compatible system mentioned above is far from being directed.

$L(\cdot, B)$ is a contravariant functor; not much happens functorially in the second variable. It transforms $l$-epimorphisms (onto maps) into o-embeddings. The functor also preserves finite cardinal sums.

If the sequence $0 \rightarrow A \rightarrow B \rightarrow C \rightarrow 0$ is exact, i.e., $C \simeq B \backslash A$, then $0 \rightarrow L(C, X) \rightarrow L(B, X) \rightarrow L(A, X)$ is exact for any o-group $X$, provided $B \rightarrow C$ is a retraction. This happens in all of the following nontrivial cases: (1) $C$ is a projective $l$-group relative to all $l$-epimorphisms; (2) $B$ is divisible and $A$ is a prime subgroup of $B$; (3) $B$ is a direct, lexicographic extension of $A$ by $C$.

1. Preliminaries. Suppose $\left\{G_{i} \mid i \in I\right\}$ is a family of p. o. groups. If $G$ is the direct sum of the $G_{i}$ we call $G$ the cardinal sum of the $G_{i}$ if we define $0 \leqq g \in G$ if and only if $0 \leqq g_{i} \in G_{i}$ for all $i \in I$; notation: $G=\boxplus\left\{G_{i} \mid i \in I\right\}$. If each $G_{i}$ is an l-group and $G$ is the cardinal sum, then $G$ is also an $l$-group. $Z$ (resp. $R$ ) denotes the additive group of integers (resp. real numbers), with the usual ordering. We observe that an Archimedean o-group is o-isomorphic to a subgroup of $\boldsymbol{R}$ in its usual order; (Hölder's theorem, [3]). A prime subgroup $N$ of the $l$-group $G$ is a convex $l$-subgroup such that $G / N$ is an ogroup. A p.o. group $G$ is semi-closed if given $g \in G$ and $n g \geqq 0$, with $n$ a positive integer, it follows that $g \geqq 0$.

We use $(\subset) \subseteq$ for (proper) containment of sets; the symbol $\backslash$ for complementation in sets.

All groups in this discussion shall be abelian. If $A$ and $B$ are $l$-groups $\mathscr{L}(A, B)$ will denote the set of $l$-homomorphisms of $A$ into 
$B$. We would like to construct a group $L(A, B)$ which "comes close" to behaving like a group of homomorphisms; the problem is of course that the sum of two l-homomorphisms need not be an $l$-homomorphism. Conrad and Diem have come up with a rather large set of $l$-endomorphisms of an l-group, which does turn out to be a semigroup under the usual addition of homomorphisms; they are the so-called $p$-endomorphisms, or polar-preserving endomorphisms (see [2]). We shall mention them in the sequel.

Suppose $A$ and $B$ are $l$-groups and $\theta, \phi \in \mathscr{L}(A, B)$. We say that $\phi$ dominates $\theta$ if $a \phi \wedge b=0$ implies $a \theta \wedge b=0$, for all $0 \leqq a \in A$ and $0 \leqq b \in B$; our notation for this is $\theta \prec \phi$. It is immediate that $\prec$ is a quasi-ordering of $\mathscr{L}(A, B)$. In fact, if $\theta \prec \phi$ and also $\phi \prec \theta$ we write $\phi \sim \theta$ and call $\phi$ and $\theta$ polar equivalent; $\sim$ is indeed an equivalence relation. Moreover, it induces a partial order on the equivalence classes, which we shall index $\left\{\mathscr{L}_{i}(A, B) \mid i \in I\right\}: \mathscr{L}_{i}(A, B) \leqq \mathscr{L}_{j}(A, B)$ if and only if some $\phi \in \mathscr{L}_{j}(A, B)$ dominates a $\theta \in \mathscr{L}_{i}(A, B)$. Now for each $i \in I$ let $L_{i}(A, B)^{+}=\left\{\theta \in \mathscr{L}(A, B) \mid \theta \prec \phi\right.$, with $\left.\phi \in \mathscr{L}_{i}(A, B)\right\}=$ $\mathrm{U}_{j \leqq i} \mathscr{L}_{i}(A, B)$. (We think of $I$ as being partially ordered so as to be compatible with the order induced on the equivalence classes.)

We are almost ready to state our first lemma; $\operatorname{Hom}(A, B)$ is of course the full homomorphism group, $\mathscr{B}(A, B)$ the subgroup of Hom $(A, B)$ generated by $\mathscr{L}(A, B)$. Thus $\mathscr{B}(A, B)=\left\{\theta_{1}-\theta_{2} \mid \theta_{1}, \theta_{2}\right.$ are sums of $l$-homomorphisms of $A$ into $B$.

Lemma 1.1. (a) For each $i \in I L_{i}(A, B)^{+}$is a subsemigroup of $\operatorname{Hom}(A, B)$; that is, if $\theta_{1}, \theta_{2} \prec \phi$ then $\theta_{1}+\theta_{2} \in \mathscr{L}(A, B)$ and $\theta_{1}+\theta_{2} \prec \phi$.

(b) For each $i \in I \mathscr{L}_{i}(A, B)$ is a subsemigroup of $L_{i}(A, B)^{+}$.

Proof. (a) Suppose $x \wedge y=0$ in $A$; then $x \phi \wedge y \phi=0$ for $\phi \in$ $\mathscr{L}(A, B)$. If $\theta_{1}, \theta_{2} \prec \phi$ we get $x \theta_{1} \wedge y \phi=0$, and in turn $x \theta_{1} \wedge y \theta_{2}=0$. Likewise $x \theta_{2} \wedge y \theta_{1}=0$, and of course $x \theta_{i} \wedge y \theta_{i}=0$ for $i=1$, 2, so that $\left(x \theta_{1}+x \theta_{2}\right) \wedge\left(y \theta_{1}+y \theta_{2}\right)=0$, and so $\theta_{1}+\theta_{2}$ is an $l$-homomorphism. If $a \phi \wedge b=0$ then $a \theta_{i} \wedge b=0$ for both $i=1,2$, so $a \theta_{1}+a \theta_{2} \wedge b=0$, which means that $\theta_{1}+\theta_{2} \prec \phi$.

(b) We check that if $\theta_{1}$ and $\theta_{2}$ are polar equivalent to $\phi$ then so is $\theta_{1}+\theta_{2}$. We already know that $\phi$ dominates $\theta_{1}+\theta_{2}$. Yet if $a \theta_{1}+a \theta_{2} \wedge b=0$ then since $0 \leqq a \theta_{1} \leqq a \theta_{1}+a \theta_{2}$ it follows that $a \theta_{1} \wedge$ $b=0$, whence $a \phi \wedge b=0$. The conclusion is that $\phi \prec \theta_{1}+\theta_{2}$, and hence $\theta_{1}+\theta_{2} \sim \phi$.

For each $i \in I$ let $L_{i}(A, B)$ be the subgroup of $\mathscr{B}(A, B)$ generated by $L_{i}(A, B)^{+}$. If we declare an element $\phi \in L_{i}(A, B)$ positive when it is an $l$-homomorphism, one easily sees that $L_{i}(A, B)$ becomes a (directed) p. o. group whose cone is $L_{i}(A, B)^{+}$. If $i \leqq j$, let $f_{i j}$ stand for the 
inclusion map of $L_{i}(A, B)$ into $L_{j}(A, B)$. We define $L(A, B)$ to be the co-limit in the category of abelian groups of the system $\left\{L_{i}(A, B) \mid\left\{f_{i j}\right\}\right\}$. (It is easily verifiable that $f_{i j}$ is the identity on $L_{i}(A, B)$, and that if $i \leqq j \leqq k$ then $f_{i j} f_{j k}=f_{i k}$.)

Proposition 1.2. $L(A, B)$ is obtained as a quotient group of the direct sum of the $L_{i}(A, B)$ by factoring out the subgroup generated by all elements of the form

$(\cdots, 0, \cdots, 0, \phi, 0, \cdots, 0,-\phi, 0, \cdots)$ (with the two nonzero entries in the $i$ th and $j$ th position respectively, and $\phi \in L_{i}(A, B)$ while $\left.i \leqq j\right)$.

Proof. The statement of the proposition merely sets out in detail the definition of a co-limit in the category of abelian groups.

Thus a typical element of $L(A, B)$ is a vector $\left(\cdots, \phi_{i}, \cdots\right)$ which is finitely nonzero, while addition and equality of vectors is subject to the identification imposed by Proposition 1.2; the entry $\phi_{i} \in L_{i}(A, B)$. The direct sum of the $L_{i}(A, B)$ may be ordered cardinally using the partial orders on the $L_{i}(A, B)$; it is clear also that the subgroup being factored out is trivially ordered in this partial order. We therefore have a partial order on $L(A, B)$ defined by $0 \leqq \phi \in L(A, B)$ if $\phi$ has a representation $\left(\cdots, \phi_{i}, \cdots\right)$ where each $\phi_{i}$ is an $l$-homomorphism.

A representation $\phi=\left(\cdots, \phi_{i}, \cdots\right)$ is said to be in reduced form if (1) for all $i \neq j$ in the support of $\left(\cdots, \phi_{i}, \cdots\right) i$ and $j$ have no common upper bound in $I$, and (2) the cardinality of the support is minimal with respect to satisfying (1). The following lemma is obvious.

LEMMA 1.3. (a) Each $\phi \in L(A, B)$ can be put in reduced form. If $\left(\cdots, \phi_{i}, \cdots\right)$ and $\left(\cdots, \theta_{i}, \cdots\right)$ are reduced forms of $\phi$, then their supports have the same cardinality, and there is a bijection $\pi$ of the supports such that $\phi_{i}=\theta_{\pi(i)}$.

(b) $0 \leqq \phi \in L(A, B)$ if and only if it has a reduced form $\left(\cdots, \phi_{i}, \cdots\right)$ such that $\phi_{i} \in \mathscr{L}(A, B)$ for each $i \in I$. If so then any reduced representation is by l-homomorphisms.

Proposition 1.4. $L(A, B)$ is a directed, semi-closed p. o. group.

Proof. $L(A, B)$ is obviously directed, so we need only verify it is semi-closed. Let $\phi \in L(A, B)$ and suppose $\left(\cdots, \phi_{i}, \cdots\right)$ is a reduced form of $\phi$. Suppose $n \phi \geqq 0$ for a positive integer $n$; the representation $\left(\cdots, n \phi_{i}, \cdots\right)$ of $n \dot{\phi}$ is clearly again in reduced form. Hence by Lemma 1.3 each $n \phi_{i}$ is an $l$-homomorphism; one can easily check that each $\phi_{i}$ is in fact an l-homomorphism. 
Proposition 1.5. If $B$ is an Archimedean l-group then $L(A, B)$ is an Archimedean p.o. group, in the sense that if $0 \leqq \phi \in L(A, B)$ and $n \theta \leqq \phi$ for each positive integer $n$, then $\theta \leqq 0$.

Proof. Suppose $n \theta \leqq \phi, \phi \geqq 0$ and $\theta=\left(\cdots, \theta_{i}, \cdots\right)$ and $\phi=$ $\left(\cdots, \phi_{i}, \cdots\right)$ are both given in reduced form. After reducing $\phi-n \theta$ we have three possibilities for an index $i$ of the support of $\phi-n \theta$ :

$$
(\phi-n \theta)_{i}= \begin{cases}\phi_{j} & \text { for some } j \in I \\ -n \theta_{k} & \text { for some } k \in I \\ \text { a sum of the above }\end{cases}
$$

Again invoking Lemma 1.3 it follows that $-n \theta_{k}$ is an $l$-homomorphism, and $\phi_{j}-n \theta_{k} \geqq 0$ for all $n=1,2, \cdots$ whenever the third choice occurs. In either case, (in the latter using the archimedeaneity of $B$ ) it follows that $\theta_{k} \leqq 0$. This shows $\theta \leqq 0$, and we are done.

For some information concerning the structure of $L(A, B)$ we look in the remainder of this section at some special cases.

$A$ is an o-group 1.6. In this situation the $l$-homomorphisms of $A$ into $B$ are simply the o-homomorphisms. The index set $I$ is then directed, since the sum of two o-homomorphisms is an o-homomorphism. $\mathscr{B}(A, B)$ then reduces to $\left\{\phi_{1}-\phi_{2} \mid \phi_{i}\right.$ are o-homomorphisms of $A$ into $B$. Since each $L_{i}(A, B)$ is a subgroup of $\mathscr{B}(A, B)$ we may take their union over $I$; it is easily seen that this union is precisely $\mathscr{B}(A, B)$. Moreover, $L(A, B)$ is now the direct limit of the $L_{i}(A, B)$; it is well known that the direct limit of subgroups of an abelian group is the union of the subgroups. Hence $\mathscr{B}(A, B) \simeq L(A, B)$.

We have a converse of sorts:

Proposition 1.6(a). Suppose $A$ is not an o-group; then there is an o-group $B$ so that the index set $I$ in the construction of $L(A, B)$ is not directed.

Proof. Suppose $A$ is not an o-group, and select $0<x, y \in A$ such that $x \wedge y=0$. Let $M$ (resp. $N$ ) be a prime subgroup that fails to contain $x$ (resp. $y$ ); then $y \in M$ and $x \in N$. Note that $A / M$ and $A / N$ are o-groups; we form $B$, the direct lexicographic extension of $A / M$ by $A / N$. We consider two $l$-homomorphisms $\phi$ and $\theta$ from $A$ into $B$ : $\phi$ is the canonical map from $A$ onto $A / M$, followed by the (convex) inclusion of $A / M$ in $B$; $\theta$ is the canonical map from $A$ onto $A / N$ followed by the inclusion of that in $B$. Now observe that 


$$
\begin{aligned}
& (\phi+\theta)(x-y) \vee 0=[(\phi+\theta) x-(\phi+\theta) y] \vee 0 \\
& =(M+x, N-y) \vee 0=0
\end{aligned}
$$

whereas

$$
(\phi+\theta)[(x-y) \vee 0]=(\phi+\theta) x=(M+x, 0)>0 .
$$

We conclude therefore that $\phi+\theta$ is not an $l$-homomorphism. The index set $I$ that arises in the construction of $L(A, B)$ is then not directed.

$B$ is an o-group 1.7. One can verify with little trouble that $\phi \in \mathscr{L}(A, B)$ dominates $\theta \in \mathscr{L}(A, B)$ if and only if $\operatorname{Ker}(\phi) \subseteq \operatorname{Ker}(\theta)$. Hence $\phi$ and $\theta$ are polar equivalent if and only if they have the same kernel. The kernels are all prime subgroups of $A$, and so $I$ is antiisomorphic to a subset of the root system of primes (see [1], Theorem 1.7, the $l$-ideals containing a prime subgroup lie on a chain). $I$ is therefore a tree-system: no two incomparable elements of $I$ have a common upper bound; plainly, $I$ is far from being directed.

Now if $\phi \in L(A, B)$ then any vector representing $\phi$ is "almost" in reduced form; that is, it satisfies the first defining condition, except the support may be too large.

$B=R$ 1.8. From the discussion in 1.7 it is clear that the index set $I$ is trivially ordered. We will show there is in fact an index $i \in I$ for each maximal $l$-ideal of $A$, and that $L(A, B)$ is a cardinal sum of copies of $\boldsymbol{R}$, one for each maximal $l$-ideal of $A$.

If $\phi: A \rightarrow B$ is an $l$-homomorphism, then $M=\operatorname{Ker}(\phi)$ is a maximal $l$-ideal. Using the fundamental theorem of $l$-homomorphisms there is an o-isomorphism $\bar{\phi}: A / M \rightarrow B$, which, by a well known corollary to Hölder's theorem, is a left multiplication by a positive real number. Thus the $l$-homomorphisms of $A$ into $B$ with kernel $M$ form a semigroup which is o-isomorphic to the additive semigroup of positive real numbers. This proves that each $L_{i}(A, B)$ is a copy of $\boldsymbol{R}$. It is clear that one such copy appears for each maximal $l$-ideal of $A$, since the corresponding quotient groups are all o-isomorphic to subgroups of $\boldsymbol{R}$.

Finally, the subgroup one factors out of the direct sum of these copies of $\boldsymbol{R}$ to get $L(A, B)$ is trivial here, and we conclude that $L(A, B)$ is a cardinal sum of copies of $\boldsymbol{R}$.

A similar argument can be made for $B=Z$; one then obtains that $L(A, B)$ is a cardinal sum of copies of $Z$, one for each maximal $l$-ideal of $A$ with cyclic factor in $A$.

A polar preserving endomorphism of an l-group $A$ is an $l$-endomorphism $\phi$ with the property that $x \wedge y=0$ in $A$ implies that $x \phi \wedge y=0$. (For an in-depth discussion of these endomorphisms the reader is 
referred to [2].) In our notation the semigroup of polar preserving endomorphisms is precisely the set of l-endomorphisms which are dominated by the identity on $A$. The subgroup they generate is one of the $L_{i}(A, A)$.

If $\phi$ is an $l$-homomorphism of $A$ onto $B$ and $\theta$ is a polar preserving endomorphism ( $p$-endomorphism) of $B$, then $\phi \theta \prec \phi$, for if $x \phi \wedge y=0$ then $x \dot{\phi} \theta \wedge y=0$. Conversely, if $\phi^{\prime} \in \mathscr{L}(A, B)$ and $\phi^{\prime} \prec \phi$ one easily sees that $\operatorname{Ker}(\phi) \subseteq \operatorname{Ker}\left(\phi^{\prime}\right)$. This implies the existence of an endomorphism $\theta$ of $B$ satisfying $b \theta=a \phi^{\prime}$ if $b=a \dot{\phi} . \quad \theta$ is certainly well defined, and it is a $p$-endomorphism since $\phi$ dominates $\phi^{\prime}$. It follows then that if $i$ is the index in $I$ determined by $\phi, L_{i}(A, B)$ is o-isomorphic to the group generated by the $p$-endomorphisms of $B$.

We close this section with a rather general comment: for arbitrary $l$-groups $A$ and $B$ the groups $L_{i}(A, B)$ are subgroups of $\mathscr{B}(A, B)$; the inclusion mappings are compatible with the $f_{i j}$, so by the definition of co-limits we have a "natural" homomorphism of $L(A, B)$ into $\mathscr{B}(A, B)$. It assigns to $\phi=\left(\cdots, \phi_{i}, \cdots\right)$ the sum of the $\phi_{i}$ in $\mathscr{B}(A, B)$. About all that is on the surface concerning this mapping is that it is onto and an o-homomorphism. As a major unanswered question we might pose the following: when is this mapping an o-isomorphism? In most of the examples one can dream up it is, but as the $l$-groups get more complex, our knowledge of the structure of $L(A, B)$ decreases rapidly.

2. The functor $L(\cdot, B)$. We will show that $L(\cdot, B)$ is a contravariant functor from the category of abelian $l$-groups and $l$-homomorphisms into the category of directed, semi-closed p. o. groups with o-homomorphisms. $(L(A, \cdot)$ does not seem to be a functor at all.)

Suppose $\phi: A \rightarrow A^{\prime}$ is an $l$-homomorphism; if $\theta_{1}, \theta_{2}: A^{\prime} \rightarrow B$ are $l$-homomorphisms and $\theta_{1} \prec \theta_{2}$ then $\phi \theta_{1} \prec \phi \theta_{2}$. Thus $\phi$ induces an ohomomorphism $\phi^{i}$ of each $L_{i}\left(A^{\prime}, B\right)$ into some $L_{\phi(i)}(A, B)$; the map $i \rightarrow \phi(i)$ is an order preserving map of $I\left(A^{\prime}, B\right)$ into $I(A, B)$. We have canonical embeddings $\mu_{i}: L_{i}(A, B) \rightarrow L(A, B)(i \in I(A, B))$ and

$$
\bar{\mu}_{j}: L_{j}\left(A^{\prime}, B\right) \longrightarrow L\left(A^{\prime}, B\right)\left(j \in I\left(A^{\prime}, B\right)\right) \text {. }
$$

We also have the connecting embeddings $\left\{f_{i j}\right\}$, for $i \leqq j \in I(A, B)$, and $\left\{\bar{f}_{i j}\right\}$, for $i \leqq j \in I\left(A^{\prime}, B\right)$. Consider now for each $i \in I\left(A^{\prime}, B\right)$ the map $\phi^{i} \mu_{\dot{\phi}(i)}: L_{i}\left(A^{\prime}, B\right) \rightarrow L(A, B)$. We show that if $i \leqq j$ in $I\left(A^{\prime}, B\right)$ then

$$
\bar{f}_{i j} \dot{\phi}^{j} \mu_{\dot{\phi}(j)}=\phi^{i} \mu_{\dot{\rho}(i)} ;
$$

for if $0 \leqq \alpha_{1}, \alpha_{2} \in L_{i}\left(A^{\prime}, B\right)$ 


$$
\begin{aligned}
\left(\alpha_{1}-\alpha_{2}\right) \bar{f}_{i j} \phi^{j} \mu_{\phi(j)}=\left(\phi \alpha_{1}-\phi \alpha_{2}\right) \mu_{\phi(j)} & =\left(\phi \alpha_{1}-\phi \alpha_{2}\right) f_{\phi(i) \phi(j)} \mu_{\phi(j)} \\
& =\left(\phi \alpha_{1}-\phi \alpha_{2}\right) \mu_{\phi(i)} \\
& =\left(\alpha_{1}-\alpha_{2}\right) \phi^{i} \mu_{\phi(i)} .
\end{aligned}
$$

By the definition of the co-limit there is a unique homomorphism $L(\phi, B): L\left(A^{\prime}, B\right) \rightarrow L(A, B)$ such that $\bar{\mu}_{i} L(\phi, B)=\phi^{i} \mu_{\phi(i)}$, for each $i \in I\left(A^{\prime}, B\right)$. Thus if

$$
\alpha=\left(\cdots, \alpha_{i}, \cdots\right) \in L\left(A^{\prime}, B\right), \quad \alpha L(\phi, B)=\left(\cdots,\left(\phi \alpha_{i}\right)_{\phi(i)}, \cdots\right) ;
$$

it is clear then that $L(\phi, B)$ is order preserving.

The next two lemmas are easy to prove; consequently we shall not bore the reader with their proofs.

LEMMA 2.1. $L(\cdot, B)$ is a contravariant functor; that is if $\phi: A_{1} \rightarrow A_{2}$ and $\theta: A_{2} \rightarrow A_{3}$ are l-homomorphisms then

$$
L(\phi \theta, B)=L(\theta, B) \cdot L(\phi, B),
$$

and $L\left(1_{A}, B\right)=1_{L(A, B)}$. $\left(1_{G}\right.$ denotes the identity mapping on $\left.G.\right)$

LEMMA 2.2. If $\phi, \phi^{\prime}: A \rightarrow A^{\prime}$ are l-homomorphisms and $\phi+\phi^{\prime}$ is too, then $L\left(\phi+\phi^{\prime}, B\right)=L(\phi, B)+L\left(\phi^{\prime}, B\right)$.

In a category $\mathscr{C}$ with zero the co-kernel of a morphism $f: A \rightarrow B$ is a morphism $\gamma: B \rightarrow C$ such that $f \gamma=0$, and having the property that if $\delta: B \rightarrow D$ is any morphism with $f \delta=0$, then there is a unique morphism $\delta^{\prime}: C \rightarrow D$ such that $\gamma \delta^{\prime}=\delta$. In the category of abelian $l$-groups the co-kernel of an $l$-homomorphism $\phi: A \rightarrow B$ is the canonical mapping $\eta: B \rightarrow B / J$ where $J$ is the convex hull of the image of $\phi$. All epimorphisms of this category have zero co-kernel, but not conversely. For instance, the embedding $j: Z \rightarrow Z \boxplus Z$ onto the diagonal has zero co-kernel, but if $\phi$ denotes the $l$-automorphism of $\boldsymbol{Z} \boxplus \boldsymbol{Z}$ given by $(a, b) \phi=(b, a)$ then $j \phi=j \cdot 1_{z \boxplus Z}=j$, so $j$ is not epic.

THEOREM 2.3. If $\alpha: A \rightarrow B$ is an l-homomorphism with zero cokernel then $L(\alpha, X)$ has a trivially ordered kernel. This holds in particular if $\alpha$ is epic. If $\alpha$ is onto $B$ then $L(\alpha, X)$ is one-to-one.

Proof. Suppose $\phi=\left(\cdots, \phi_{i}, \cdots\right) \in L(B, X)$ with each $\phi_{i} \geqq 0$, and assume $\phi L(\alpha, X)=0$. Thus $\left(\cdots,\left(\alpha \phi_{i}\right)_{\alpha(i)}, \cdots\right)=0$; this means that the vector $\left(\cdots,\left(\alpha \phi_{i}\right)_{\alpha(i)}, \cdots\right)$ of $\boxplus\left\{L_{i}(A, X) \mid i \in I(A, X)\right\}$ is in a trivially ordered subgroup. Thus each $\alpha \phi_{i}=0$, and since $\alpha$ has zero cokernel, each $\phi_{i}=0$.

Now suppose $\alpha$ is onto and $\theta \in L(B, X)$. If $\theta=\left(\cdots, \theta_{i}, \cdots\right)$ is in 
reduced form then we seek to show $\left(\cdots,\left(\alpha \theta_{i}\right)_{\alpha(i)}, \cdots\right)$ is too. Clearly $i \in I(B, X)$ is in the support of $\left(\cdots, \theta_{i}, \cdots\right)$ if and only if $\alpha(i)$ is in the support of $\left(\cdots,\left(\alpha \theta_{i}\right)_{\alpha(i)}, \cdots\right)$ since $\alpha$ is onto. Suppose now that $\alpha(i), \alpha(j)$ are both in the support of $\left(\cdots,\left(\alpha \theta_{i}\right)_{\alpha(i)}, \cdots\right)$ and $k \in I(A, X)$ exceeds both of them. Then whenever $0 \leqq \gamma_{i} \in L_{i}(B, X)$ and $0 \leqq \gamma_{j} \in$ $L_{j}(B, X), \alpha \gamma_{i}+\alpha \gamma_{j}=\alpha\left(\gamma_{i}+\gamma_{j}\right)$ is an $l$-homomorphism of $A$ into $X$. Again using the fact that $\alpha$ is onto one can then readily show that $\gamma_{i}+\gamma_{j}$ is an $l$-homomorphism. But then some index of $I(B, X)$ exceeds $i$ and $j$, contradicting the hypothesis that $\left(\cdots, \theta_{i}, \cdots\right)$ is reduced. A similar argument shows that the size of the support of $\left(\cdots,\left(\alpha \theta_{i}\right)_{\alpha(i)}, \cdots\right)$ is minimal; it now follows that $\left(\cdots,\left(\alpha \theta_{i}\right)_{\alpha(i)}, \cdots\right)$ is reduced.

Thus if $0=\theta L(\alpha, X)=\left(\cdots,\left(\alpha \theta_{i}\right)_{\alpha(i)}, \cdots\right)$ then each $\alpha \theta_{i}=0$ and so $\theta_{i}=0$ for all $i \in I(B, X)$; hence $\theta=0$ and so $L(\alpha, X)$ is one-to-one. (We shall see later that $L(\alpha, X)$ is in fact an o-embedding.)

The natural question here is: what does $L(\cdot, X)$ do to short exact sequences of l-groups? (We call a sequence $0 \rightarrow A \stackrel{\alpha}{\rightarrow} B \stackrel{\beta}{\rightarrow} C \rightarrow 0$ of $l$-homomorphisms short exact if $\alpha$ is one to one, $\beta$ is onto and $\operatorname{Ker}(\beta)=\operatorname{Im}(\alpha)$.) We will show presently that $L(\beta, X)$ is an omonomorphism. Certainly $L(\beta, X) \cdot L(\alpha, X)=L(\alpha \beta)=$,0 , but do we get exactness at $L(B, X)$ ? We shall give some partial answers, and then make some (hopefully) educated guesses.

Proposition 2.4. If $0 \rightarrow A \stackrel{\alpha}{\rightarrow} B \stackrel{\beta}{\rightarrow} C \rightarrow 0$ is a short exact sequence of l-groups, and if $0 \leqq \phi \in \operatorname{Ker}(L(\alpha, X))$ then $\phi \in\left(L(C, X)^{+}\right) L(\beta, X)$. In particular $L(\beta, X)$ is an o-embedding.

Proof. If $\phi=\left(\cdots, \phi_{i}, \cdots\right) \geqq 0$ and $\phi L(\alpha, X)=0$ then

$$
\left(\cdots,\left(\alpha_{\phi_{i}}\right)_{\alpha(i)}, \cdots\right)=0 \text {. }
$$

This means that in $\boxplus\left\{L_{i}(A, X) \mid i \in I(A, X)\left\{\left(\cdots,\left(\alpha \phi_{i}\right)_{\alpha(i)}, \cdots\right)\right.\right.$ is a vector whose components add to zero. But each entry $\alpha \phi_{i}$ is 0 or an $l$-homomorphism; if the sum of $l$-homomorphisms is zero each of them is zero. Thus $\alpha \phi_{i}=0$ for each $i \in I(B, X)$; since $\beta$ is the co-kernel of $\alpha$, there is an l-homomorphism $\gamma^{i}: C \rightarrow X$ such that $\beta \gamma^{i}=\phi_{i}$. This determines a $\gamma \in L(C, X)$ whose image under $L(\beta, X)$ is $\dot{\phi}$; clearly $0 \leqq \gamma$ and our proposition is proved.

Proposition 2.5. If $0 \rightarrow A \stackrel{\alpha}{\rightarrow} B \stackrel{\beta}{\rightarrow} C \rightarrow 0$ splits cardinally, i.e. $B \cong A \boxplus C$, then $L(B, X) \cong L(C, X) \boxplus L(A, X)$.

Proof. If $B \cong A \boxplus C$ we have $l$-homomorphisms $\rho: C \rightarrow B$ and $\sigma: B \rightarrow A$ such that $\alpha \sigma=1_{A}, \rho \beta=1_{C}, \rho \sigma=0$ and $1_{B}=\sigma \alpha+\beta \rho$. For 
each $l$-group $X$ we have

$$
\begin{array}{ll}
L(\sigma, X) L(\alpha, X)=1_{L(A, X)}, & L(\beta, X) L(\rho, X)=1_{L(C, X),}, \\
L(\sigma, X) L(\rho, X)=0, & L(\beta, X) L(\alpha, X)=0,
\end{array}
$$

and finally by Lemma 2.2

$$
L(\alpha, X) L(\sigma, X)+L(\rho, X) L(\beta, X)=1_{L(B, X)} \cdot
$$

This proves $L(B, X) \cong L(C, X) \boxplus L(A, X)$.

Proposition 2.6. Let $j: G \rightarrow \bar{G}$ be the natural embedding of the l-group $G$ in its divisible hull. For each l-group $X L(j, X)$ is an o-embedding. If $X$ is divisible $L(j, X)$ is onto.

Proof. If $\phi_{1}$ and $\phi_{2}$ are any two homomorphisms of $\bar{G}$ into the $l$-group $X$ which agree on $G$, then since each $x \in \bar{G}$ is of the form $x=(1 / n) g$, for a suitable positive integer $n$, we have

$$
n\left(x \dot{\phi}_{1}\right)=n((1 / n) g) \phi_{1}=g \dot{\phi}_{1}=g \phi_{2}=n((1 / n) g) \phi_{2}=n\left(x \phi_{2}\right),
$$

which implies that $x \phi_{1}=x \phi_{2}$, since $X$ is torsion free. Clearly then $L(j, X)$ is one-to-one. Moreover, if $\phi: \bar{G} \rightarrow X$ is a homomorphism whose restriction to $G$ is an $l$-homomorphism then $\phi$ is an $l$-homomorphism; for if $x=(1 / n) g \in \bar{G}$ with $g \in G$ then

$$
\begin{gathered}
n(x \vee 0) \phi=n((1 / n) g \vee 0) \phi=(g \vee 0) \phi=g \phi \vee 0=[n(1 / n) g \phi] \vee 0 \\
=n[(1 / n) g \phi \vee 0]=n(x \phi \vee 0) .
\end{gathered}
$$

This says that $L(j, X)$ is an o-embedding. Finally, if $X$ is divisible then each $l$-homomorphism of $G \rightarrow X$ extends (uniquely) to an $l$-homomorphism of $\bar{G} \rightarrow X$; in other words, $L(j, X)$ is onto.

We shall for the remainder of the section study the question of exactness of $L(\cdot, X)$ for o-groups $X$; according to 1.7 the picture we get of $L(A, X)$ is somewhat less cluttered. The preceding result tells us that if $X$ is divisible we might as well assume that $A$ is. So we ask: given an o-group $X$, which exact sequences $0 \rightarrow A \rightarrow B \rightarrow C \rightarrow 0$ go to exact sequences

$$
0 \rightarrow L(C, X) \rightarrow L(B, X) \rightarrow L(A, X) ?
$$

Prior to going into these questions more deeply we record some interesting properties of $L(\cdot, X)$.

Proposition 2.7. Let $\phi: A \rightarrow B$ be an l-homomorphism onto $B$. If $L(\phi, X)$ is an o-isomorphism for each o-group $X$ then $\phi$ itself is an isomorphism. 
REMARK. An analogous statement holds for o-groups $X$ with a minimal nonzero convex subgroup.

Proof. If $\phi$ is not one-to-one pick $0<x \in \operatorname{Ker}(\phi)$ and let $N$ be a prime subgroup that fails to contain $x$. Set $X=A / N$ and $\eta: A \rightarrow X$ to be the canonical $l$-homomorphism. Then $(\cdots, 0, \cdots, \eta, \cdots, 0, \cdots) \in$ $L(A, X)$ is not an image under $L(\phi, X)$.

THEOREM 2.8. Let $A$ be an l-group; $A$ is a subdirect product of reals if and only if whenever $\phi: A \rightarrow B$ is an l-homomorphism onto $B$ then $L(\phi, R): L(B, R) \rightarrow L(A, R)$ is an l-isomorphism if and only if $\phi$ is an l-isomorphism.

Proof. Suppose $\phi: A \rightarrow B$ is an l-homomorphism onto $B$. Let us examine what $L(\phi, R)$ does. There is a one-to-one correspondence between the maximal $l$-ideals of $B$ and the maximal $l$-ideals of $A$ that contain $K=\operatorname{Ker}(\phi)$. Now $L(B, R)$ and $L(A, R)$ are both cardinal sums of copies of $\boldsymbol{R}$, one for each maximal $l$-ideal of $B$ and $A$ respectively. So $L(\phi, R)$ is nothing more than the injection of $L(B, R)$ onto that portion of $L(A, R)$ corresponding to maximal $l$-ideals of $A$ that contain $K$.

If $L(\phi, R)$ is then onto for some $\phi$ with nonzero kernel, then every maximal $l$-ideal of $A$ contains $K$ and so $A$ is not a subdirect product of reals. Conversely, if $A$ is not a subdirect product of reals let $D$ be the intersection of all the maximal $l$-ideals of $A ; D \neq 0$. Let $B=A / D$ and $\phi$ be the canonical mapping of $A$ onto $B$. By our arguments in the previous paragraph $L(\phi, R)$ is an $l$-isomorphism.

REMARK. A similar theorem holds for subdirect products of integers.

THEOREM 2.9. Let $0 \rightarrow A \stackrel{\alpha}{\rightarrow} B \stackrel{\beta}{\rightarrow} C \rightarrow 0$ be a short exact sequence of l-groups. If $X$ is any Archimedean o-group then the induced sequence

$$
0 \rightarrow(C, X) \rightarrow L(B, X) \rightarrow L(A, X) \quad \text { is exact. }
$$

If $X=\boldsymbol{R}$ then $L(\alpha, X)$ is onto if and only if every maximal l-ideal of $A$ is the meet of a maximal l-ideal of $B$ with $A$. If this is the case $L(B, X) \cong L(C, X) \boxplus L(A, X)$. If $X=Z$ then $L(\alpha, X)$ is onto if and only if every maximal l-ideal of $A$ with cyclic factor is the meet with $A$ of a maximal l-ideal of $B$ with cyclic factor.

Proof. As in 1.8 we have that if $\phi: B \rightarrow X$ is an l-homomorphism its kernel $M$ is a maximal $l$-ideal and $\phi$ determines an o-isomorphism 
from $B / M \rightarrow X$ which is a right multiplication by a suitable positive real number. The difference here is that not all maximal $l$-ideals appear as indices for $L_{i}(B, X)$, and the $L_{i}(B, X)$ themselves need not be full copies of $\boldsymbol{R}$. Still $L(B, X)$ is a cardinal sum of subgroups of $\boldsymbol{R}$ one for each "admissible" maximal $l$-ideal. Now $L(\beta, X)$ acts as in the proof of 2.8: there still is a one-to-one correspondence between maximal $l$-ideals of $C$ that appear as kernels of $l$-homomorphisms into $X$ and the same type of maximal $l$-ideals of $B$ that contain $A$. So $L(\beta, X)$ is the injection of $L(C, X)$ onto that portion of $L(B, X)$ corresponding to those maximal $l$-ideals of $B$ that contain $A$.

As for $L(\alpha, X)$ we have the following: if $\phi: B \rightarrow X$ is once again an $l$-homomorphism, and $M=\operatorname{Ker}(\phi) \nsupseteq A$ then $M \cap A$ is a maximal $l$-ideal of $A$ and it is the kernel of $\alpha \phi$. Thus $L(\alpha, X)$ has the effect of annihilating all the components of $L(B, X)$ corresponding to maximal $l$-ideals of $B$ that contain $A$, and being the identity on the remaining components.

It is now clear that $0 \rightarrow L(C, X) \rightarrow L(B, X) \rightarrow L(A, X)$ is exact, and also that the last part of the theorem holds, in the special cases when $X=\boldsymbol{R}$ or $X=Z$.

In fact, after we record the following definition we have a better theorem.

Let $X$ be an o-group and $G$ be any $l$-group; a prime subgroup $N$ of $G$ is an $X$-entry of $G$ if it appears as the kernel of some $l$-homomorphism of $G$ into $X$. Thus:

THEOREM 2.9a. If $0 \rightarrow A \stackrel{\alpha}{\rightarrow} B \stackrel{\beta}{\rightarrow} C \rightarrow 0$ is exact then $L(B, X) \cong$ $L(C, X) \boxplus L(A, X)$ for an Archimedean o-group $X$ if and only if every $X$-entry of $A$ is the meet of an $X$-entry of $B$ with $A$.

We have the following sufficient condition for the exactness of $0 \rightarrow L(C, X) \rightarrow L(B, X) \rightarrow L(A, X)$, when $X$ is an arbitrary o-group.

TheOREm 2.10. If $0 \rightarrow A \stackrel{\alpha}{\rightarrow} B \stackrel{\beta}{\rightarrow} C \rightarrow 0$ is exact, then $0 \rightarrow L(C, X) \rightarrow$ $L(B, X) \rightarrow L(A, X)$ is exact if $A+N=B$ for every $X$-entry of $B$ which does not contain $A$.

The proof of this theorem depends upon the following lemma, which is known and quite easy to prove. (See [1], Theorem 1.14.)

Lemma 2.11. Let $G$ be an l-group, $A$ be a nonzero l-ideal of $G$. There is an o-isomorphism between the set of prime subgroups of $G$ that do not contain $A$ and the proper prime subgroups of $A$ via the mapping $N \mapsto N \cap A$. 
Proof of 2.10. Since the index sets $I(\cdot, X)$ are inversely o-isomorphic to a subset of prime subgroups we shall use the prime subgroups themselves to index the groups that make up the $L(\cdot, X)$ 's.

Suppose then that $\phi=\left(\cdots, \phi_{N}, \cdots\right)$ is in reduced form and $\phi L(\alpha, X)=0$, that is $\left(\cdots,\left(\alpha \phi_{N}\right)_{N \cap A}, \cdots\right)=0$. If $N \supseteqq A$ then $\alpha \phi_{N}$ is identically zero; to see this write $\phi_{N}=\phi_{N}^{+}-\phi_{N}^{-}$, with $\phi_{N}^{+}, \phi_{N}^{-} \in \mathscr{L}(B, X)$; the kernels of $\phi_{N}^{+}$and $\phi_{N}^{-}$contain $N$ and hence $A$. In this case we need not worry about $\phi_{N}$; pick $\theta, \psi \in L(C, X)$ such that $\beta \theta=\phi_{N}^{+}$and $\beta \psi=\phi_{N}^{-}$; then $\beta(\theta-\psi)=\phi_{N}$.

We are therefore left to consider those prime subgroups $N$ of $B$ which do not contain $A$. By Lemma 2.11 the support of $\left(\cdots,\left(\alpha \phi_{N}\right)_{N \cap A}, \cdots\right)$ is determined by precisely those prime subgroups; the lemma also guarantees that the representation is reduced. We have then that $\alpha \dot{\phi}_{N}=0$ for each prime subgroup $N \nsupseteq A$. Once again, writing $\phi_{N}=\phi_{N}^{+}-\phi_{N}^{-}$ as a difference of $l$-homomorphisms (whose kernels contain $N$ but not $A$, for otherwise they would also vanish when restricted to $A$ ) we have $\alpha \dot{\phi}_{N}^{+}=\alpha \dot{\phi}_{N}^{-}$.

Our assumption is though that $A+N=B$ for each such prime subgroup $N$, and this implies that $\phi_{N}^{+}=\phi_{\bar{N}}^{-}$. The conclusion here is that the support of $\phi=\left(\cdots, \dot{\phi}_{N}, \cdots\right)$ consists of those $X$-entries which contain $A$. Our first paragraph in this proof then makes it clear that $\phi$ is the image of some element of $L(C, X)$ under $L(\beta, X)$. This completes the proof of the theorem.

Corollary 2.10.1. Suppose $A$ is a maximal l-ideal of $B$, let $C=B / A$ and $0 \rightarrow A \rightarrow B \rightarrow C \rightarrow 0$ be the induced exact sequence. If $A$ is also a minimal prime subgroup then $0 \rightarrow L(C, X) \rightarrow L(B, X) \rightarrow$ $L(A, X)$ is exact for all o-groups $X$.

An l-group $G$ is hyper-archimedean if it is Archimedean and every $l$-homomorphic image of $G$ is Archimedean. It is well known (see for instance [1], Theorem 2.4) that $G$ is hyper-archimedean if and only if every prime subgroup is maximal (and hence minimal).

COROLlaRy 2.10.2. If $B$ is a hyper-archimedean l-group and $0 \rightarrow A \rightarrow B \rightarrow C \rightarrow 0$ is exact then $0 \rightarrow L(C, X) \rightarrow L(B, X) \rightarrow L(A, X)$ is exact for every o-group $X$.

Proof. Every prime subgroup of $B$ is both maximal and minimal; consequently, if $N$ is an $X$-entry of $B$ that does not contain $A$ we have $B=A+N$. Theorem 2.10 now applies.

Another sufficient condition for the exactness of $0 \rightarrow L(C, X) \rightarrow$ 
$L(B, X) \rightarrow L(A, X)$ is obtained by requiring that $0 \rightarrow A \stackrel{\alpha}{\rightarrow} B \stackrel{\beta}{\rightarrow} C \rightarrow 0$ be "right splitting", i.e., that $\beta$ be a retract.

THEOREM 2.11. Let $0 \rightarrow A \stackrel{\alpha}{\rightarrow} B \stackrel{\beta}{\rightarrow} C \rightarrow 0$ be an exact sequence of l-groups, and suppose $\rho: C \rightarrow B$ is an l-homomorphism such that $\rho \beta=1_{c}$. Then for each o-group $X 0 \rightarrow L(C, X) \rightarrow L(B, X) \rightarrow L(A, X)$ is exact.

Proof. We use the notation of the proof of Theorem 2.10. Let $\phi=\left(\cdots, \phi_{N}, \cdots\right)$ be an element of $L(B, X)$ in reduced form and consider $\phi L(\alpha, X)=\left(\cdots,\left(\alpha \phi_{N}\right)_{N \cap A}, \cdots\right)$; as shown in 2.10 this is once again reduced. So if $\phi L(\alpha, X)=0$ we have $\alpha \phi_{N}=0$ for all $X$-entries $N$ of $B$. As before, write $\phi_{N}=\phi_{N}^{+}-\phi_{N}^{-}$as the difference of $l$-homomorphisms of $B$ into $X$. For each $X$-entry define $\theta_{N}^{+}, \theta_{N}^{-}: C \rightarrow X$ by $\theta_{N}^{+}=\rho \phi_{N}^{+}$and $\theta_{N}^{-}=\rho \phi_{N}^{-}$. We claim that $\theta L(\beta, X)=\phi$, where $\theta=$ $\left(\cdots, \theta_{N}, \cdots\right)$ and $\theta_{N}=\theta_{N}^{+}-\theta_{N}^{-}$.

Note that $\rho$ induces a group direct $\operatorname{sum} B \cong A \oplus C$; more precisely, each $b \in B$ can be expressed uniquely as $b=a \alpha+c \rho$, where $c=b \beta$. Thus $b \beta \theta_{N}^{+}=b \beta \rho \phi_{N}^{+}$and $b \beta \theta_{N}^{-}=b \beta \rho \phi_{N}^{-}$, while $b \phi_{N}^{+}=a \alpha \phi_{N}^{+}+c \rho \phi_{N}^{+}=$ $a \alpha \phi_{N}^{+}+b \beta \rho \phi_{N}^{+}=a \alpha \phi_{N}^{+}+b \beta \theta_{N}^{+}$; likewise $b \phi_{N}^{-}=a \alpha \phi_{\bar{N}}+b \beta \theta_{N}^{-}$, which implies that $b \phi_{N}=b \beta \theta_{N}$, for all $b \in B$.

This suffices to prove that $\theta L(\beta, X)=\dot{\phi}$, and our theorem is proved.

CoRollary 2.11.1. Let $0 \rightarrow A \stackrel{\alpha}{\rightarrow} B \stackrel{\beta}{\rightarrow} C \rightarrow 0$ be an exact sequence; in all of the cases below $0 \rightarrow L(C, X) \rightarrow L(B, X) \rightarrow L(A, X)$ is exact for each o-group $X$.

(a) $C$ is a projective l-group.

(b) $B$ is divisible and $A$ is a prime subgroup of $B$.

(c) $B$ is a direct lexicographic extension of $A$ by $C$.

Proof. In each of the above cases $\beta$ is a retract and the theorem applies.

Corollary 2.11.2. If $0 \rightarrow A \rightarrow B \rightarrow C \rightarrow 0$ is exact where $A$ is a prime subgroup of $B$ then $0 \rightarrow L(C, X) \rightarrow L(B, X) \rightarrow L(A, X)$ is exact for each divisible o-group $X$.

Proof. Apply Proposition 2.6 and Corollary 2.11.1 (b).

The following example may serve to illustrate a bit the difficulty in deciding which conjectures ought to be made in connection with this functor. Let $X=Z \overrightarrow{\times} Z$ with the lexicographic order: that is, 
$(m, n) \geqq 0$ if $m>0$ or $m=0$ and then $n \geqq 0$. We will show that if $0 \rightarrow A \rightarrow B \rightarrow C \rightarrow 0$ is a short exact sequence then $0 \rightarrow L(C, X) \rightarrow$ $L(B, X) \rightarrow L(A, X)$ is exact. So consider an exact sequence $0 \rightarrow A \rightarrow$ $B \rightarrow C \rightarrow 0$, suppose $\phi=\left(\cdots, \phi_{N}, \cdots\right)$ is in reduced form and $\phi L(\alpha, X)=0(\phi \in L(B, X))$. As in the proof of 2.10 it suffices to consider those $X$-entries $N$ such that $N \nsupseteq A$. As before write $\phi_{N}=$ $\phi_{N}^{+}-\phi_{N}^{-}$as a difference of $l$-homomorphisms whose kernels do not contain $A$. By our assumption $\alpha \phi_{N}^{+}=\alpha \phi_{N}^{-} ; \phi_{N}^{+}$and $\phi_{N}^{-}$have a common kernel, and after factoring out this kernel we have two o-embeddings of $X$ into itself, say $\theta_{1}$ and $\theta_{2}$, which agree on the nonzero proper convex subgroup of $X$. The o-homomorphisms of $X$ into itself are given by triangular integral matrices

$$
\left(\begin{array}{cc}
m & p \\
0 & n
\end{array}\right) \text { with } m>0, n \geqq 0 \text { or } m=n=0 \text { and } p \geqq 0 \text {. }
$$

If $\theta_{i}=\left(\begin{array}{cc}m_{i} & p_{i} \\ 0 & n_{i}\end{array}\right)(i=1,2)$ and $\theta_{1}$ agrees with $\theta_{2}$ as specified, then $n_{1}=n_{2}$, so clearly $\theta_{1}-\theta_{2}$ is either order preserving or order inverting.

Lifting back to $B \phi_{N}^{+}-\phi_{N}^{-}$is either an l-homomorphism or the additive inverse of one. Since $\alpha\left(\phi_{N}^{+}-\phi_{N}^{-}\right)=0$ there is a unique $l$ homomorphism $\psi: C \rightarrow X$ such that $\beta \psi= \pm\left(\phi_{N}^{+}-\phi_{N}^{-}\right)$. This suffices to prove the exactness of the sequence.

The reader will appreciate the special nature of the above example.

3. Comments and questions. It appears that our functor will be of little use as the classical Hom-functor is in extension theory of abelian groups and modules. One might try to define an Ext-like functor using projective resolutions; in that case the question of independence of the resolution used appears to be an impossible problem. Or one could choose some "standard" free resolution; here it is obvious that computations could become nightmarish.

In view of some of our results, particularly Theorems 2.8 and 2.9 , one can expect $L(\cdot, X)$ to be useful in characterizing certain lattice-group theoretical concepts. In any case, one large disadvantage of our construction is that there is no functoriality in the second variable.

Another possibility is that $L(\cdot, R)$ might serve as a "duality" functor between $l$-groups and abelian groups. Then one practically has to restrict oneself to subdirect products of reals, $(L(A, R)=0$ if $A$ has no maximal $l$-ideals), and then two such subdirect products of reals might very well have the same dual, (if they have the same number of maximal $l$-ideals.) A true duality can be realized, at least 
for subdirect products of reals, if one computes $L(A, X)$ for every Archimedean o-group, and then associates for each $A$ the whole "spectrum" $\{L(A, X) \mid X$ is a subgroup of $R\}$. Such a duality is evidently too cumbersome.

\section{REFERENCES}

1. P. Conrad, Lattice-Ordered Groups, Tulane University, 1970.

2. P. Conrad and J. Diem, The ring of polar-preserving endomorphisms of an abelian lattice-ordered group; Illinois J. Math., 15, No. 2, (1971), 222-240.

3. L. Fuchs, Partially Ordered Algebraic Systems, Pergamon Press, 1963.

4. B. Mitchell, Theory of Categories, Academic Press, 1965.

Received June 19, 1972.

UNIVERSITY OF FLORIDA 



\section{PACIFIC JOURNAL OF MATHEMATICS}

\section{EDITORS}

RICHARD ARENS (Managing Editor)

University of California

Los Angeles, California 90024

R. A. Beaumont

University of Washington

Seattle, Washington 98105
J. DUGUNDJI*

Department of Mathematics

University of Southern California

Los Angeles, California 90007

D. Gilbarg and J. Milgram

Stanford University

Stanford, California 94305

\section{ASSOCIATE EDITORS}
E. F. BECKENBACH
B. H. NeumanN
F. WOLF
K. YosHIDA

\section{SUPPORTING INSTITUTIONS}

UNIVERSITY OF BRITISH COLUMBIA
CALIFORNIA INSTITUTE OF TECHNOLOGY
UNIVERSITY OF CALIFORNIA
MONTANA STATE UNIVERSITY
UNIVERSITY OF NEVADA
NEW MEXICO STATE UNIVERSITY
OREGON STATE UNIVERSITY
UNIVERSITY OF OREGON
OSAKA UNIVERSITY

UNIVERSITY OF BRITISH COLUMBIA

UNIVERSITY OF CALIFORNIA

MONTANA STATE UNIVERSITY

UNIVERSITY OF NEVADA

OREGON STATE UNIVERSITY

OSAKA UNIVERSITY
UNIVERSITY OF SOUTHERN CALIFORNIA

STANFORD UNIVERSITY

UNIVERSITY OF TOKYO

UNIVERSITY OF UTAH

WASHINGTON STATE UNIVERSITY

UNIVERSITY OF WASHINGTON

\section{AMERICAN MATHEMATICAL SOCIETY} NAVAL WEAPONS CENTER

* C. R. DePrima California Institute of Technology, Pasadena, CA 91109, will replace J. Dugundji until August 1974. 


\section{Pacific Journal of Mathematics}

\section{Vol. 48, No. $1 \quad$ March, 1973}

Jan Aarts and David John Lutzer, Pseudo-completeness and the product of Baire

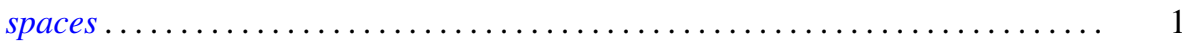

Gordon Owen Berg, Metric characterizations of Euclidean spaces ............ 11

Ajit Kaur Chilana, The space of bounded sequences with the mixed topology ..... . 29

Philip Throop Church and James Timourian, Differentiable open maps of

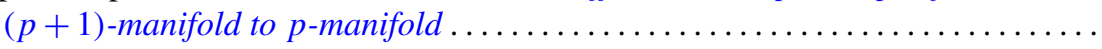

P. D. T. A. Elliott, On additive functions whose limiting distributions possess a finite

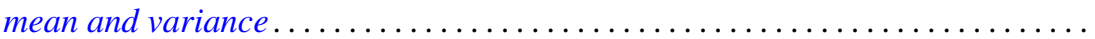

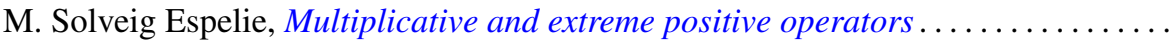

Jacques A. Ferland, Domains of negativity and application to generalized convexity

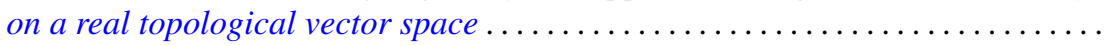

Michael Benton Freeman and Reese Harvey, A compact set that is locally holomorphically convex but not holomorphically convex ...............

Roe William Goodman, Positive-definite distributions and intertwining

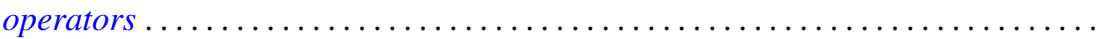

Elliot Charles Gootman, The type of some $C^{*}$ and $W^{*}$-algebras associated with

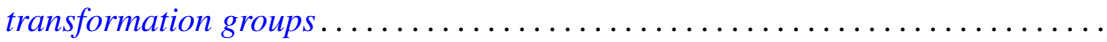

David Charles Haddad, Angular limits of locally finitely valent holomorphic

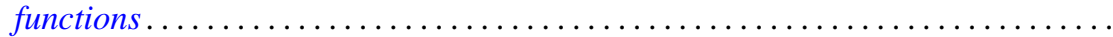

William Buhmann Johnson, On quasi-complements .

William M. Kantor, On 2-transitive collineation groups of finite projective spaces...

Joachim Lambek and Gerhard O. Michler, Completions and classical localizations of right Noetherian rings

Kenneth Lamar Lange, Borel sets of probability measures ......

David Lowell Lovelady, Product integrals for an ordinary differential equation in a Banach space

Jorge Martinez, A hom-functor for lattice-ordered groups .........

W. K. Mason, Weakly almost periodic homeomorphisms of the two sphere ....

Anthony G. Mucci, Limits for martingale-like sequences .......

Eugene Michael Norris, Relationally induced semigroups ...

Arthur E. Olson, A comparison of c-density and $k$-density ......

Donald Steven Passman, On the semisimplicity of group rings of linear groups.

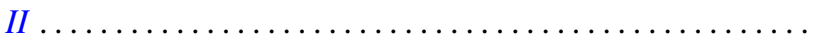

Charles Radin, Ergodicity in von Neumann algebras .

P. Rosenthal, On the singularities of the function generated by the Bergman operator of the second kind.

Arthur Argyle Sagle and J. R. Schumi, Multiplications on homogeneous spaces,

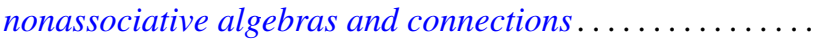

Leo Sario and Cecilia Wang, Existence of Dirichlet finite biharmonic functions on

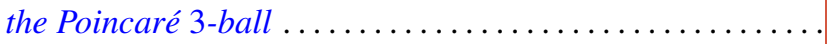

Ramachandran Subramanian, On a generalization of martingales due to Blake ..

Bui An Ton, On strongly nonlinear elliptic variational inequalities.

Seth Warner, A topological characterization of complete, discretely valued

fields. 